\title{
HAUSDORFF TOPOLOGY INDUCED BY THE FUZZY METRIC AND THE FIXED POINT THEOREMS IN FUZZY METRIC SPACES
}

\author{
HSien-Chung Wu
}

\begin{abstract}
The Hausdorff topology induced by a fuzzy metric space under more weak assumptions is investigated in this paper. Another purpose of this paper is to obtain the Banach contraction theorem in fuzzy metric space based on a natural concept of Cauchy sequence in fuzzy metric space.
\end{abstract}

\section{Introduction and motivation}

Let $X$ be a universal set. For any $x, y \in X$, let $\tilde{d}(x, y)$ be a fuzzy subset of $\mathbb{R}_{+}$with membership function $\xi_{\tilde{d}(x, y)}: \mathbb{R}_{+} \rightarrow[0,1]$, where the value $\xi_{\tilde{d}(x, y)}(t)$ means that the membership degree of the distance between $x$ and $y$ is equal to $t$. Therefore we can define a function $M: X \times X \times[0, \infty) \rightarrow[0,1]$ given by

$$
M^{*}(x, y, t)=\xi_{\tilde{d}(x, y)}(t) .
$$

The fuzzy metric space based on the concept in (1) was proposed by Kaleva and Seikkala [10]. On the other hand, inspired by the Menger space which is a special kind of probabilistic metric space by referring to Schweizer and Sklar [12, 13, 14], Hadžić and Pap [9] and Chang et al. [2], Kramosil and Michalek [11] proposed another concept of fuzzy metric space. In this paper, we shall consider the weaker axioms than that of the concept proposed by Kramosil and Michalek [11]. The formal definition is given below.

Definition 1.1. Let $X$ be a nonempty universal set, let $*$ be a t-norm, and let $M$ be a mapping defined on $X \times X \times[0, \infty)$ into [0,1]. The 3 -tuple $(X, M, *)$ is called a fuzzy metric space if and only if the following conditions are satisfied:

- for any $x, y \in X, M(x, y, t)=1$ for all $t>0$ if and only if $x=y$;

- $M(x, y, 0)=0$ for all $x, y \in X$;

- $M(x, y, t)=M(y, x, t)$ for all $x, y \in X$ and $t \geq 0$;

Received January 14, 2015; Revised May 11, 2015.

2010 Mathematics Subject Classification. 54E35, 54H25.

Key words and phrases. Banach contraction theorem, contractive mapping, fuzzy metric space, Hausdorff topology, triangular norm. 
- $M(x, y, t) * M(y, z, s) \leq M(x, z, t+s)$ for all $x, y, z \in X$ and $s, t \geq 0$ (the so-called triangle inequality).

The mapping $M$ in fuzzy metric space $(X, M, *)$ can be regarded as a membership function of a fuzzy subset of $X \times X \times[0, \infty)$. Sometimes $M$ is called a fuzzy metric of the space $(X, M, *)$. According to the first and second conditions of fuzzy metric space, the mapping $M(x, y, t)$ can be interpreted as the membership degree of the distance that is less than or equal to $t$ between $x$ and $y$. Therefore, the meanings of $M$ and $M^{*}$ defined in (1) are different.

The following assumption

$$
M(x, y, t)>0 \text { for } t>0
$$

was taken by many researchers (e.g. George and Veeramani [3, 4], Gregori and Romaguera [6] and Gregori and Sapena [8]), which is too strong in some sense. The reason is that, if $t>0$ is very small, then $M(x, y, t)$ can be zero. George and Veeramani [3] took the assumption (2) and claimed that the fuzzy metric space can induce a Hausdorff topology by further assuming that the t-norm and fuzzy metric $M$ are continuous. In this paper, we shall not consider the assumption (2), and assume that the fuzzy metric $M$ and the t-norm are just left-continuous: the assumption which are weaker than that of George and Veeramani [3]. Under these weak assumptions, we can show that this fuzzy metric space can also induce a Hausdorff topology.

On the other hand, Grabiec [5] provided a fixed point theorem in fuzzy metric space by defining a Cauchy sequence $\left\{x_{n}\right\}_{n \in \mathbb{N}}$ to be satisfying

$$
\lim _{n \rightarrow \infty} M\left(x_{n+p}, x_{n}, t\right)=1
$$

for all $p>0$ and $t>0$, and said that the fuzzy metric space is complete if every Cauchy sequence defined in this sense is convergent. Subrahmanyam [17] also used this concept to study the common fixed point theorem in fuzzy metric spaces. However, George and Veeramani $[3,4]$ pointed out that this kind of definition of Cauchy sequence is inappropriate by providing that even $\mathbb{R}$ fails to be complete. In other words, the fixed point theorem in fuzzy metric space provided by Grabiec [5] is inappropriate. Alaca et al. [1] and Sharma and Deshpande [15] also adopted the concept of Cauchy sequence defined in (3) to investigate the fixed point theorem in intuitionistic fuzzy metric spaces. The reasonable concept of Cauchy sequence was proposed by George and Veeramani $[3,4]$ in which $\left\{x_{n}\right\}_{n \in \mathbb{N}}$ is a Cauchy sequence if and only if, given any $t>0$ and $0<r<1$, there exists $n_{r, t} \in \mathbb{N}$ such that $M\left(x_{m}, x_{n}, t\right)>1-r$ for all $m, n \geq n_{r, t}$. The different concepts of Cauchy sequence in Definition 3.2 below were also studied by Song [16], Vasuki and Veeramani [18] and Gregori and Sapena [8]. In this paper, we shall provide the Banach contraction theorem in fuzzy metric space based on the natural concept of Cauchy sequence given in Definition 3.2

This paper is organized as follows. In Section 2, we induce a Hausdorff topology from a given fuzzy metric space under the weak assumptions as we 
mentioned above. In Section 4, the Banach contraction theorem in fuzzy metric space is obtained under the natural concept of Cauchy sequence in Definition 3.2 .

\section{Hausdorff topology}

We first recall the concept of a t-norm. The function $*:[0,1] \times[0,1] \rightarrow[0,1]$ that satisfies the following axioms is called a t-norm (triangular norm):

- (boundary condition) $a * 1=a$;

- (commutativity) $a * b=b * a$;

- (increasing property) if $b \leq c$, then $a * b \leq a * c$;

- (associativity) $(a * b) * c=a *(b * c)$.

Remark 2.1. We have the following useful observations.

- By the commutativity of t-norm, if the t-norm is continuous with respect to the first component (resp. second component), then it is also continuous with respect to the second component (resp. first component). In other words, for any fixed $a \in[0,1]$, if the function $f(x)=a * x$ (resp. $f(x)=x * a$ ) is continuous, then the function $g(x)=x * a$ (resp. $g(x)=a * x)$ is continuous. Similarly, if the t-norm is left-continuous (resp. right-continuous) with respect to the first or second component, then it is also left-continuous (resp. right-continuous) with respect to each component.

- The associativity of t-norm says that we can consider the operations

$$
a_{1} * a_{2} * \cdots * a_{p}
$$

for $p \geq 2$. Also, the commutativity of t-norm says that if the t-norm is continuous (resp. left-continuous or right-continuous) with respect any one of the component, then it is also continuous (resp. left-continuous or right-continuous) with respect to each component.

The following useful results can be obtained by routine argument.

Proposition 2.1. Suppose that the t-norm $*$ is left-continuous at 1 with respect to the first or second component. Then

(i) for any $a, b \in(0,1)$ with $a>b$, there exists $r \in(0,1)$ such that $a * r \geq b$;

(ii) for any $a \in(0,1)$ and any $n \in \mathbb{N}$ with $n \geq 2$, there exists $r \in(0,1)$ such that $r * r * \cdots * r>$ a for $n$-times.

In the sequel, we shall induce the Hausdorff topology from a given fuzzy metric space. Here, we shall not assume that the mapping $M(x, y, \cdot):(0, \infty) \rightarrow$ $[0,1]$ and the t-norm $*$ are continuous. The following results will be useful for further discussion.

Proposition 2.2. Let $(X, M, *)$ be a fuzzy metric space. Given any fixed $x, y \in X$, the mapping $M(x, y, \cdot):(0, \infty) \rightarrow[0,1]$ has the following properties:

(i) The mapping $M(x, y, \cdot)$ is nondecreasing; 
(ii) If the mapping $M(x, y, \cdot)$ is left-continuous and $M(x, y, t)>1-r$, then there exists $t_{0}$ with $0<t_{0}<t$ such that $M\left(x, y, t_{0}\right)>1-r$.

Proof. Part (i) is obvious. To prove part (ii), let $\epsilon=M(x, y, t)-(1-r)>0$. By the left-continuity, there exists $t_{0}$ with $0<t_{0}<t$ such that $\mid M(x, y, t)-$ $M\left(x, y, t_{0}\right) \mid<\epsilon$. From part (i), we also have $0 \leq M(x, y, t)-M\left(x, y, t_{0}\right)<\epsilon$, which implies $M\left(x, y, t_{0}\right)>1-r$. This completes the proof.

We remark that Proposition 2.2 improves George and Veeramani [3, Remark $2.6]$ in which the continuity of a t-norm as well as the mapping $M(x, y, \cdot)$ : $(0, \infty) \rightarrow[0,1]$ were assumed.

Definition 2.1. Let $(X, M, *)$ be a fuzzy metric space. Given $t>0$ and $0<r<1$, the $(r, t)$-neighborhood of $x$ is defined by

$$
B(x, r, t)=\{y \in X: M(x, y, t)>1-r\} .
$$

We see that $B(x, r, t) \neq \emptyset$, since $x \in B(x, r, t)$ by the fact of $M(x, x, t)=1$ for all $t>0$. Since $0<r<1$, it is obvious that if $M(x, y, t)=0$, then $y \notin B(x, r, t)$. In other words, if $y \in B(x, r, t)$, then $M(x, y, t)>0$. Let $\mathcal{B}$ denote the family of all $(r, t)$-neighborhoods of each point of $X$. The following interesting results will be used to induce a Hausdorff topology.

Proposition 2.3. Let $(X, M, *)$ be a fuzzy metric space such that the following conditions are satisfied:

- the mapping $M(x, y, \cdot):(0, \infty) \rightarrow[0,1]$ is left-continuous;

- the t-norm * is left-continuous at 1 with respect to the first or second component.

Then the family $\mathcal{B}$ of neighborhoods satisfies the following properties:

(i) For each $x \in X$, there exists $B \in \mathcal{B}$ such that $B$ is an $(r, t)$-neighborhood of $x$;

(ii) If $B(x, r, t)$ is a $(r, t)$-neighborhood of $x$ and $y \in B(x, r, t)$, then there exists $B(y, \bar{r}, \bar{t})$ such that $B(y, \bar{r}, \bar{t}) \subseteq B(x, r, t)$;

(iii) If $B\left(x_{1}, r_{1}, t_{1}\right), B\left(x_{2}, r_{2}, t_{2}\right) \in \mathcal{B}$ and $x \in B\left(x_{1}, r_{1}, t_{1}\right) \cap B\left(x_{2}, r_{2}, t_{2}\right)$, then there exists $B\left(x, r_{3}, t_{3}\right)$ such that

$$
B\left(x, r_{3}, t_{3}\right) \subseteq B\left(x_{1}, r_{1}, t_{1}\right) \cap B\left(x_{2}, r_{2}, t_{2}\right) ;
$$

(iv) If $B(x, r, t)$ is a $(r, t)$-neighborhood of $x$, there exists $n \in \mathbb{N}$ such that $B\left(x, \frac{1}{n}, \frac{1}{n}\right) \subseteq B(x, r, t)$;

(v) If $x \neq y$, then there exist disjoint neighborhoods $B\left(x, r_{1}, t_{1}\right)$ and $B\left(y, r_{2}\right.$, $\left.t_{2}\right)$.

Proof. Since $x \in B(x, r, t)$, part (i) is obvious. To prove part (ii), for $y \in$ $B(x, r, t)$, we have $M(x, y, t)>1-r$. By part (ii) of Proposition 2.2, there exists $t_{0}$ with $0<t_{0}<t$ such that $M\left(x, y, t_{0}\right)>1-r$. Let $r_{0}=M\left(x, y, t_{0}\right)$. Then we have $r_{0}>1-r$. There exists $s$ with $0<s<1$ such that $r_{0}>1-s>1-r$. By part (i) of Proposition 2.1, there exists $r_{1}$ with $0<r_{1}<1$ such that 
$r_{0} * r_{1} \geq 1-s$. Let $\bar{r}=1-r_{1}$ and $\bar{t}=t-t_{0}$. We claim that $B(y, \bar{r}, \bar{t}) \subseteq B(x, r, t)$. For $z \in B(y, \bar{r}, \bar{t})$, i.e.,

$$
M(y, z, \bar{t})=M\left(y, z, t-t_{0}\right)>1-\bar{r}=r_{1},
$$

by the property of triangle inequality, we have

$$
\begin{aligned}
M(x, z, t) & \geq M\left(x, y, t_{0}\right) * M\left(y, z, t-t_{0}\right)=r_{0} * M\left(y, z, t-t_{0}\right) \\
& \geq r_{0} * r_{1} \geq 1-s>1-r .
\end{aligned}
$$

This shows that $z \in B(x, r, t)$. Therefore we obtain the desired inclusion.

To prove part (iii), using part (ii), there exist $\bar{t}_{1}, \bar{t}_{2}, \bar{r}_{1}, \bar{r}_{2}$ such that

$$
B\left(x, \bar{r}_{1}, \bar{t}_{1}\right) \subseteq B\left(x_{1}, r_{1}, t_{1}\right) \text { and } B\left(x, \bar{r}_{2}, \bar{t}_{2}\right) \subseteq B\left(x_{2}, r_{2}, t_{2}\right) .
$$

We take $t_{3}=\min \left\{\bar{t}_{1}, \bar{t}_{2}\right\}$ and $r_{3}=\min \left\{\bar{r}_{1}, \bar{r}_{2}\right\}$. Then, for $y \in B\left(x, r_{3}, t_{3}\right)$, using part (i) of Proposition 2.2, we have

$$
M\left(x, y, \bar{t}_{1}\right) \geq M\left(x, y, t_{3}\right)>1-r_{3} \geq 1-\bar{r}_{1}
$$

and

$$
M\left(x, y, \bar{t}_{2}\right) \geq M\left(x, y, t_{3}\right)>1-r_{3} \geq 1-\bar{r}_{2},
$$

which says that

$$
y \in B\left(x, \bar{r}_{1}, \bar{t}_{1}\right) \cap B\left(x, \bar{r}_{2}, \bar{t}_{2}\right) \subseteq B\left(x_{1}, r_{1}, t_{1}\right) \cap B\left(x_{2}, r_{2}, t_{2}\right) .
$$

Therefore we obtain the desired inclusion.

To prove part (iv), we take $n \in \mathbb{N}$ such that $\frac{1}{n} \leq \min \{r, t\}$. Then, for $y \in B\left(x, \frac{1}{n}, \frac{1}{n}\right)$, we have

$$
M(x, y, t) \geq M\left(x, y, \frac{1}{n}\right)>1-\frac{1}{n} \geq 1-r,
$$

which says that $y \in B(x, r, t)$.

To prove part $(\mathrm{v})$, since $x \neq y$, by the definition of fuzzy metric space, there exists $t_{0}>0$ such that $M\left(x, y, t_{0}\right)<1$. There also exists $r_{0}$ such that $M\left(x, y, t_{0}\right)<r_{0}<1$. By part (ii) of Proposition 2.1, there exists $\hat{r}$ with $0<\hat{r}<1$ such that $\hat{r} * \hat{r}>r_{0}$. Now we consider $B\left(x, 1-\hat{r}, \frac{t_{0}}{2}\right)$ and $B\left(y, 1-\hat{r}, \frac{t_{0}}{2}\right)$. We claim that $B\left(x, 1-\hat{r}, \frac{t_{0}}{2}\right) \cap B\left(y, 1-\hat{r}, \frac{t_{0}}{2}\right)=\emptyset$ by contradiction. Suppose that $z \in B\left(x, 1-\hat{r}, \frac{t_{0}}{2}\right) \cap B\left(y, 1-\hat{r}, \frac{t_{0}}{2}\right)$. Then, by the property of triangle inequality and the symmetry, we have

$$
M\left(x, y, t_{0}\right) \geq M\left(x, z, \frac{t_{0}}{2}\right) * M\left(z, y, \frac{t_{0}}{2}\right) \geq \hat{r} * \hat{r}>r_{0}>M\left(x, y, t_{0}\right),
$$

which is a contradiction. The proof is complete.

Theorem 2.1. Let $(X, M, *)$ be a fuzzy metric space such that the following conditions are satisfied:

- the mapping $M(x, y, \cdot):(0, \infty) \rightarrow[0,1]$ is left-continuous;

- the t-norm * is left-continuous at 1 with respect to the first or second component. 
Then the family $\mathcal{B}$ of neighborhoods induces a Hausdorff topology $\tau$ such that $\mathcal{B}$ is a base for $\tau$ and $\tau$ also satisfies the first axiom of countability, in which $O \in \tau$ if and only if, for each $x \in O$, there exist $t>0$ and $r \in(0,1)$ such that $B(x, r, t) \subseteq O$.

Proof. Parts (i)-(iii) of Proposition 2.3 say that if we define

$$
\begin{gathered}
\tau=\{O \subseteq X: x \in O \text { if and only if there exist } t>0 \text { and } r \in(0,1) \\
\text { such that } B(x, r, t) \subseteq O\},
\end{gathered}
$$

then $\tau$ is a topology such that $\mathcal{B}$ is a base for $\tau$. Part (iv) of Proposition 2.3 says that there exists a countable local base at each point $x \in X$, which also says that $\tau$ satisfies the first axiom of countability. Finally, part (v) of Proposition 2.3 says that $\tau$ is a Hausdorff topology. This completes the proof.

Let $(X, d)$ be a metric space. If the sequence $\left\{x_{n}\right\}_{n \in \mathbb{N}}$ in $(X, d)$ is convergent to $x$, i.e., $d\left(x_{n}, x\right) \rightarrow 0$ as $n \rightarrow \infty$, then it is denoted by $x_{n} \stackrel{d}{\longrightarrow} x$ as $n \rightarrow \infty$.

Definition 2.2. Let $(X, M, *)$ be a fuzzy metric space. A sequence $\left\{x_{n}\right\}_{n \in \mathbb{N}}$ in $X$ is said to converge to $x \in X$ if and only if

$$
\lim _{n \rightarrow \infty} M\left(x_{n}, x, t\right)=1
$$

for all $t>0$. In this case, we write $x_{n} \stackrel{M}{\longrightarrow} x$ as $n \rightarrow \infty$.

Let $(X, \tau)$ be a topological space. The convergence of a sequence $\left\{x_{n}\right\}_{n \in \mathbb{N}}$ in $X$ to $x \in X$ with respect to the topology is denoted by $x_{n} \stackrel{{ }_{\tau}}{\longrightarrow} x$ as $n \rightarrow \infty$. If $\tau$ is a Hausdorff topology, then the limit is unique. Let $(X, M, *, \tau)$ be a fuzzy metric space endowed with a Hausdorff topology $\tau$ given in Theorem 2.1. Let $\left\{x_{n}\right\}_{n \in \mathbb{N}}$ be a sequence in $X$. Since $\mathcal{B}$ is a local base for $\tau$, it follows that $x_{n} \stackrel{\tau}{\longrightarrow} x$ as $n \rightarrow \infty$, if and only if, given any $t>0$ and $0<r<1$, there exists $n_{r, t}$ such that $x_{n} \in B(x, r, t)$ for all $n \geq n_{r, t}$. Since $x_{n} \in B(x, r, t)$ means $M\left(x_{n}, x, t\right)>1-r$, it says that $x_{n} \stackrel{\tau}{\longrightarrow} x$ as $n \rightarrow \infty$, if and only if, given any $t>0$ and $0<r<1$, there exists $n_{r, t}$ such that $M\left(x_{n}, x, t\right)>1-r$ for all $n \geq n_{r, t}$.

Proposition 2.4. Let $(X, M, *)$ be a fuzzy metric space such that the following conditions are satisfied:

- the mapping $M(x, y, \cdot):(0, \infty) \rightarrow[0,1]$ is left-continuous;

- the t-norm * is left-continuous at 1 with respect to the first or second component.

Let $\tau$ be the Hausdorff topology induced by the fuzzy metric space $(X, M, *)$, and let $\left\{x_{n}\right\}_{n \in \mathbb{N}}$ be a sequence in $X$. Then $x_{n} \stackrel{\tau}{\longrightarrow} x$ as $n \rightarrow \infty$ if and only if $x_{n} \stackrel{M}{\longrightarrow} x$ as $n \rightarrow \infty$.

Proof. Suppose that $x_{n} \stackrel{\tau}{\longrightarrow} x$ as $n \rightarrow \infty$. Fixed $t>0$, given any $r \in(0,1)$, there exists $n_{r, t} \in \mathbb{N}$ such that $x_{n} \in B(x, r, t)$ for all $n \geq n_{r, t}$. This says that 
$M\left(x_{n}, x, t\right)>1-r$, i.e., $1-M\left(x_{n}, x, t\right)<r$ for all $n \geq n_{r, t}$. Since $r$ can be any positive small number, we must have $M\left(x_{n}, x, t\right) \rightarrow 1$ as $n \rightarrow \infty$. Conversely, given any $t>0$, if $M\left(x_{n}, x, t\right) \rightarrow 1$ as $n \rightarrow \infty$, then, given any $r \in(0,1)$, there exists $n_{r, t} \in \mathbb{N}$ such that $1-M\left(x_{n}, x, t\right)<r$, i.e., $M\left(x_{n}, x, t\right)>1-r$ for all $n \geq n_{r, t}$, which says that $x_{n} \in B(x, r, t)$ for all $n \geq n_{r, t}$. This shows that $x_{n} \stackrel{\tau}{\longrightarrow} x$ as $n \rightarrow \infty$, and the proof is complete.

Let $\tau$ be the Hausdorff topology induced by the fuzzy metric space $(X, M, *)$. We consider the following set

$$
\bar{B}(x, r, t)=\{y \in X: M(x, y, t) \geq 1-r\} .
$$

George and Veeramani [3, Lemma 3.16] proved $\operatorname{cl}(\bar{B}(x, r, t))=\bar{B}(x, r, t)$ under the continuity assumption on the mapping $M(x, y, \cdot):(0, \infty) \rightarrow[0,1]$ and the t-norm $*$. Now we can obtain the same result when the t-norm $*$ is assumed to be left-continuous at 1 with respect to the first or second component by modifying their proof.

Proposition 2.5. Let $(X, M, *)$ be a fuzzy metric space such that the following conditions are satisfied:

- the mapping $M(x, y, \cdot):(0, \infty) \rightarrow[0,1]$ is continuous;

- the t-norm * is left-continuous at 1 with respect to the first or second component.

Let $\tau$ be the Hausdorff topology induced by the fuzzy metric space $(X, M, *)$. Then the set

$$
\bar{B}(x, r, t)=\{y \in X: M(x, y, t) \geq 1-r\}
$$

is closed with respect to $\tau$. In other words, we have $\operatorname{cl}(\bar{B}(x, r, t))=\bar{B}(x, r, t)$.

Proof. For $y \in \operatorname{cl}(\bar{B}(x, r, t))$, since $(X, \tau)$ satisfies the first axiom of countability, there exists a sequence $\left\{y_{n}\right\}_{n \in \mathbb{N}}$ in $\bar{B}(x, r, t)$ such that $y_{n} \stackrel{\tau}{\longrightarrow} y$ as $n \rightarrow \infty$. By Proposition 2.4, we have $M\left(y_{n}, y, t\right) \rightarrow 1$ as $n \rightarrow \infty$ for all $t>0$. Since $M\left(y_{n}, y, t\right) \leq 1$ for all $n$, it says that $\left\{M\left(y_{n}, y, t\right)\right\}_{n \in \mathbb{N}}$ is a bounded sequence. Therefore, there exists a subsequence $\left\{M\left(y_{n_{k}}, y, t\right)\right\}_{n_{k} \in \mathbb{N}}$ such that $M\left(y_{n_{k}}, y, t\right) \uparrow 1$ as $n_{k} \rightarrow \infty$. Given any $\epsilon>0$, the property of triangle inequality says that

$$
M(x, y, t+\epsilon) \geq M\left(x, y_{n_{k}}, t\right) * M\left(y_{n_{k}}, y, \epsilon\right) \geq(1-r) * M\left(y_{n_{k}}, y, \epsilon\right) .
$$

From Remark 2.1, we see that the t-norm $*$ is left-continuous at 1 with respect to each component. Therefore we obtain

$$
M(x, y, t+\epsilon) \geq(1-r) *\left(\lim _{n_{k} \rightarrow \infty} M\left(y_{n_{k}}, y, \epsilon\right)\right)=(1-r) * 1=1-r .
$$

By the right-continuity of the mapping $M(x, y, \cdot):(0, \infty) \rightarrow[0,1]$, we also have

$$
M(x, y, t)=\lim _{\epsilon \rightarrow 0+} M(x, y, t+\epsilon) \geq 1-r,
$$

which says that $y \in \bar{B}(x, r, t)$, and the proof is complete. 


\section{Fixed point theorems}

Since $M(x, y, t)$ is the membership degree of the distance that is less than or equal to $t$ between $x$ and $y$, the mapping $M(x, y, \cdot)$ is nondecreasing as shown in part (i) of Proposition 2.2. Therefore, intuitively, if $t$ is sufficiently small, then the membership degree $M(x, y, t)$ is close to 0 . In other words, we expect to have $M(x, y, t) \rightarrow 0$ as $t \rightarrow 0+$. Therefore we propose the following definition.

Definition 3.1. The 3 -tuple $(X, M, *)$ is called a rational fuzzy metric space if and only if $(X, M, *)$ is a fuzzy metric space and satisfies the following property:

$$
\lim _{t \rightarrow 0+} M(x, y, t)=0
$$

for any fixed $x, y \in X$ with $x \neq y$.

Let $(X, M, *)$ be a fuzzy metric space. Given any fixed $x, y \in X$ with $x \neq y$ and $\lambda \in[0,1)$, we define

$$
\Theta_{\lambda}(x, y)=\{t>0: M(x, y, t) \leq 1-\lambda\}
$$

and a function $\Lambda_{\lambda}: X \times X \rightarrow[0,+\infty)$ by

$$
\Lambda_{\lambda}(x, y)=\sup \Theta_{\lambda}(x, y)=\sup \{t>0: M(x, y, t) \leq 1-\lambda\} .
$$

For convenience, we put $\Lambda_{\lambda}(x, x)=0$ for all $\lambda \in[0,1)$.

Proposition 3.1. Let $(X, M, *)$ be a rational fuzzy metric space. Then

$$
\Theta_{\lambda}(x, y) \neq \emptyset \text { for } x \neq y \text {. }
$$

Proof. Suppose that $\Theta_{\lambda}(x, y)=\emptyset$. Then we must have $M(x, y, t)>1-\lambda$ for all $t>0$. Therefore we obtain

$$
\lim _{t \rightarrow 0+} M(x, y, t) \geq 1-\lambda>0,
$$

which contradicts the fact that $(X, M, *)$ is a rational fuzzy metric space.

If $(X, M, *)$ is a rational fuzzy metric space, then we see that

$$
\Lambda_{0}(x, y)=\sup \{t>0: M(x, y, t) \leq 1\}=\sup \{t>0\}=+\infty .
$$

Proposition 3.2. Let $(X, M, *)$ be a rational fuzzy metric space. Given any fixed $x, y \in X$ with $x \neq y$, the following statements hold true.

(i) If $\Lambda_{\lambda}(x, y)=+\infty$, then $M(x, y, t) \leq 1-\lambda$ for all $t>0$.

(ii) We have $\Lambda_{\lambda}(x, y)>0$ for all $\lambda \in(0,1)$.

Proof. To prove part (i), if $\Lambda_{\lambda}(x, y)=+\infty$, then $M(x, y, t) \leq 1-\lambda$ for sufficiently large $t>0$. Suppose that there exists $t_{0}>0$ such that $M\left(x, y, t_{0}\right)>$ $1-\lambda$. Since $M(x, y, \cdot)$ is nondecreasing, if $t_{1}>t_{0}$, then $M\left(x, y, t_{1}\right)>1-\lambda$, which contradicts the fact of $M(x, y, t) \leq 1-\lambda$ for sufficiently large $t>0$.

To prove part (ii), given any $\epsilon>0$, suppose that $M\left(x, y, \Lambda_{\lambda}(x, y)+\epsilon\right) \leq 1-\lambda$. By definition of $\Lambda_{\lambda}$, we have $\Lambda_{\lambda}(x, y) \geq \Lambda_{\lambda}(x, y)+\epsilon$, which is a contradiction. 
Therefore we must have $M\left(x, y, \Lambda_{\lambda}(x, y)+\epsilon\right)>1-\lambda$. Now we assume that $\Lambda_{\lambda}(x, y)=0$ for $\lambda \in(0,1)$. Since $\epsilon$ can be any positive real number, we have

$$
0=\lim _{\epsilon \rightarrow 0+} M(x, y, \epsilon) \geq 1-\lambda
$$

which leads to a contradiction.

Proposition 3.3. Let $(X, M, *)$ be a rational fuzzy metric space. Given any fixed $x, y \in X$ and $\lambda \in(0,1)$, the following statements hold true.

(i) If $\Lambda_{\lambda}(x, y)<+\infty$, then for any $\epsilon>0$, we have

$$
M\left(x, y, \Lambda_{\lambda}(x, y)+\epsilon\right)>1-\lambda .
$$

Moreover, if $\epsilon>0$ is sufficiently small such that $\Lambda_{\lambda}(x, y)>\epsilon$, then

$$
M\left(x, y, \Lambda_{\lambda}(x, y)-\epsilon\right) \leq 1-\lambda .
$$

(ii) If $\Lambda_{\lambda}(x, y)<+\infty$ and $t>\Lambda_{\lambda}(x, y)$, then $M(x, y, t)>1-\lambda$. If $t<$ $\Lambda_{\lambda}(x, y)$, then $M(x, y, t) \leq 1-\lambda$.

(iii) If $M(x, y, t) \leq 1-\lambda$, then $t \leq \Lambda_{\lambda}(x, y)$. If $M(x, y, t)>1-\lambda$, then $\Lambda_{\lambda}(x, y)<+\infty$ and $t \geq \Lambda_{\lambda}(x, y)$.

Proof. To prove part (i), if $x=y$, then the inequality (4) holds true. If $x \neq y$, then the inequality (4) can be realized from the proof of part (ii) of Proposition 3.2. On the other hand, by the concept of supremum, given any $\epsilon>0$ with $\Lambda_{\lambda}(x, y)>\epsilon$, there exists $t_{\epsilon}>0$ such that $M\left(x, y, t_{\epsilon}\right) \leq 1-\lambda$ and $t_{\epsilon}>\Lambda_{\lambda}(x, y)-\epsilon$. Since the mapping $M(x, y, \cdot)$ is nondecreasing, we have

$$
M\left(x, y, \Lambda_{\lambda}(x, y)-\epsilon\right) \leq M\left(x, y, t_{\epsilon}\right) \leq 1-\lambda .
$$

To prove the first property of part (ii), if $x=y$, then the desired result is obvious. Therefore we assume $x \neq y$. If $t>\Lambda_{\lambda}(x, y)$, then there exists $\epsilon>0$ such that $t \geq \Lambda_{\lambda}(x, y)+\epsilon$. Using the nondecreasing property of the mapping $M(x, y, \cdot)$ and $(4)$, we also obtain

$$
M(x, y, t) \geq M\left(x, y, \Lambda_{\lambda}(x, y)+\epsilon\right)>1-\lambda .
$$

To prove the second property of part (ii), if $t<\Lambda_{\lambda}(x, y)$, there exists $\epsilon>0$ such that $0 \leq t \leq \Lambda_{\lambda}(x, y)-\epsilon$. Using (5), we obtain

$$
M(x, y, t) \leq M\left(x, y, \Lambda_{\lambda}(x, y)-\epsilon\right) \leq 1-\lambda .
$$

To prove the first property of part (iii), assume that $\Lambda_{\lambda}(x, y)=+\infty$. It is obvious that if $M(x, y, t) \leq 1-\lambda$, then $t \leq \Lambda_{\lambda}(x, y)$. Assume that $\Lambda_{\lambda}(x, y)<$ $+\infty$. Using the contraposition of first property of part (ii), we also have that if $M(x, y, t) \leq 1-\lambda$, then $t \leq \Lambda_{\lambda}(x, y)$. To prove the second property of part (iii), if $x=y$, then we are done. Assume that $x \neq y$ and $\Lambda_{\lambda}(x, y)=+\infty$. It says that $M(x, y, t) \leq 1-\lambda$ for sufficiently large $t$. Since $M(x, y, \cdot)$ is nondecreasing, we must have $M(x, y, t) \leq 1-\lambda$ for all $t>0$. Therefore, if $M(x, y, t)>1-\lambda$, then we must have $\Lambda_{\lambda}(x, y)<+\infty$. In this case, the contraposition of second property of part (ii) will lead to the desired result. 
Definition 3.2. Let $(X, M, *)$ be a fuzzy metric space, and let $\left\{x_{n}\right\}_{n \in \mathbb{N}}$ be a sequence in $X$.

- We say that $\left\{x_{n}\right\}_{n \in \mathbb{N}}$ is a Cauchy sequence if and only if, given any $t>0$ and $0<r<1$, there exists $n_{r, t} \in \mathbb{N}$ such that $M\left(x_{m}, x_{n}, t\right)>1-r$ for all $m, n \geq n_{r, t}$. The fuzzy metric space is called complete if and only if each Cauchy sequence is convergent.

- For any fixed $\lambda$, we say that $\left\{x_{n}\right\}_{n \in \mathbb{N}}$ is a Cauchy sequence with respect to $\Lambda_{\lambda}$ if and only if, given any $\epsilon>0$, there exists $n_{\epsilon} \in \mathbb{N}$ such that $m, n \geq n_{\epsilon}$ implies $\Lambda_{\lambda}\left(x_{m}, x_{n}\right)<\epsilon$.

Proposition 3.4. Let $(X, M, *)$ be a rational fuzzy metric space.

(i) The sequence $\left\{x_{n}\right\}_{n \in \mathbb{N}}$ in $X$ converges to $x$ if and only if $\Lambda_{\lambda}\left(x_{n}, x\right) \rightarrow 0$ as $n \rightarrow \infty$ for all $\lambda \in(0,1)$.

(ii) The sequence $\left\{x_{n}\right\}_{n \in \mathbb{N}}$ in $X$ is a Cauchy sequence if and only if it is a Cauchy sequence with respect to $\Lambda_{\lambda}$ for all $\lambda \in(0,1)$.

Proof. To prove part (i), suppose that $M\left(x_{n}, x, t\right) \rightarrow 1$ as $n \rightarrow \infty$ for all $t>0$, which says that, given any $t>0$ and $\delta>0$, there exists $n_{t, \delta} \in \mathbb{N}$ such that $\left|M\left(x_{n}, x, t\right)-1\right|<\delta$ for $n \geq n_{t, \delta}$. In other words, for any fixed $\lambda \in(0,1)$, given any $\epsilon>0$, there exists $n_{\epsilon} \in \mathbb{N}$ such that $\left|M\left(x_{n}, x, \epsilon / 2\right)-1\right|<\lambda$ for $n \geq n_{\epsilon}$, which says that $M\left(x_{n}, x, \epsilon / 2\right)>1-\lambda$ for $n \geq n_{\epsilon}$. By part (iii) of Proposition 3.3, we obtain

$$
\Lambda_{\lambda}\left(x_{n}, x\right) \leq \frac{\epsilon}{2}<\epsilon
$$

for $n \geq n_{\epsilon}$. This shows that $\Lambda_{\lambda}\left(x_{n}, x\right) \rightarrow 0$ as $n \rightarrow \infty$. For the converse, suppose that $\Lambda_{\lambda}\left(x_{n}, x\right) \rightarrow 0$ as $n \rightarrow \infty$ for all $\lambda \in(0,1)$. Given any $\delta>0$ and $\lambda \in(0,1]$, there exists $n_{\delta, \lambda} \in \mathbb{N}$ such that $\left|\Lambda_{\lambda}\left(x_{n}, x\right)\right|<\delta$ for all $n \geq n_{\delta, \lambda}$. In other words, for any fixed $t>0$, given any $\epsilon \in(0,1)$, there exists $n_{\epsilon} \in \mathbb{N}$ such that

$$
\Lambda_{\epsilon}\left(x_{n}, x\right)=\left|\Lambda_{\epsilon}\left(x_{n}, x\right)\right|<t
$$

for $n \geq n_{\epsilon}$, which says that $M\left(x_{n}, x, t\right)>1-\epsilon$ for $n \geq n_{\epsilon}$ by part (ii) of Proposition 3.3. This shows that $M\left(x_{n}, x, t\right) \rightarrow 1$ as $n \rightarrow \infty$ for all $t>0$.

To prove part (ii), suppose that $\left\{x_{n}\right\}_{n \in \mathbb{N}}$ is a Cauchy sequence, i.e., given any $t>0$ and $\delta>0$, there exists $n_{t, \delta} \in \mathbb{N}$ such that $m, n \geq n_{t, \delta}$ implies $M\left(x_{m}, x_{n}, t\right)>1-\delta$. In other words, for any fixed $\lambda \in(0,1)$, given any $\epsilon>0$, there exists $n_{\epsilon} \in \mathbb{N}$ such that $m, n \geq n_{\epsilon}$ implies $M\left(x_{m}, x_{n}, \epsilon / 2\right)>1-\lambda$. By part (iii) of Proposition 3.3, we obtain

$$
\Lambda_{\lambda}\left(x_{m}, x_{n}\right) \leq \frac{\epsilon}{2}<\epsilon
$$

for $m, n \geq n_{t, \delta}$. This shows that $\left\{x_{n}\right\}_{n \in \mathbb{N}}$ is a Cauchy sequence with respect to $\Lambda_{\lambda}$. For the converse, suppose that $\left\{x_{n}\right\}_{n \in \mathbb{N}}$ is a Cauchy sequence with respect to $\Lambda_{\lambda}$ for all $\lambda \in(0,1)$. Given any $\delta>0$ and $\lambda \in(0,1)$, there exists $n_{\delta, \lambda} \in \mathbb{N}$ such that $m, n \geq n_{\delta, \lambda}$ implies $\Lambda_{\lambda}\left(x_{m}, x_{n}\right)<\delta$. In other words, for any fixed $t>0$, given any $\epsilon \in(0,1)$, there exists $n_{\epsilon} \in \mathbb{N}$ such that $m, n \geq n_{\epsilon}$ implies 
$\Lambda_{\epsilon}\left(x_{m}, x_{n}\right)<t$, which says that $M\left(x_{m}, x_{n}, t\right)>1-\epsilon$ for $n \geq n_{\epsilon}$ by part (ii) of Proposition 3.3. This shows that $\left\{x_{n}\right\}_{n \in \mathbb{N}}$ is a Cauchy sequence, and the proof is complete.

Proposition 3.5. Let $(X, M, *)$ be a rational fuzzy metric space. We have the following properties.

(i) Suppose that the t-norm is left-continuous at 1 in the first or second component. Given any fixed $x_{1}, x_{2}, \ldots, x_{p} \in X$ and $\mu \in(0,1)$, there exists $\lambda \in(0,1)$ such that

$$
\Lambda_{\mu}\left(x_{1}, x_{p}\right) \leq \Lambda_{\lambda}\left(x_{1}, x_{2}\right)+\Lambda_{\lambda}\left(x_{2}, x_{3}\right)+\cdots+\Lambda_{\lambda}\left(x_{p-1}, x_{p}\right) .
$$

(ii) Given any fixed $x, y \in X$, if $\mu<\lambda$, then $\Theta_{\lambda}(x, y) \subseteq \Theta_{\mu}(x, y)$ and $\Lambda_{\mu}(x, y) \geq \Lambda_{\lambda}(x, y)$.

Proof. According to part (ii) of Proposition 2.1, there exists $\lambda \in(0,1)$ such that

$$
(1-\lambda) * \cdots *(1-\lambda)>1-\mu .
$$

Suppose that $\Lambda_{\lambda}\left(x_{i}, x_{i+1}\right)<+\infty$ for all $i=1, \ldots, p-1$. Given any $\epsilon>0$, we have

$$
\begin{aligned}
& M\left(x_{1}, x_{p}, \Lambda_{\lambda}\left(x_{1}, x_{2}\right)+\Lambda_{\lambda}\left(x_{2}, x_{3}\right)+\cdots+\Lambda_{\lambda}\left(x_{p-1}, x_{p}\right)+(p-1) \epsilon\right) \\
\geq & M\left(x_{1}, x_{2}, \Lambda_{\lambda}\left(x_{1}, x_{2}\right)+\epsilon\right) * \cdots * M\left(x_{p-1}, x_{p}, \Lambda_{\lambda}\left(x_{p-1}, x_{p}\right)+\epsilon\right) \\
\geq & (1-\lambda) * \cdots *(1-\lambda) \\
> & 1-\mu .
\end{aligned}
$$

The first inequality holds by the triangle inequality for t-norm $*$, the second one by (4) and the monotonicity of $*$, and the last one by (7).

To prove part (i), we consider the following cases.

- Suppose that $\Lambda_{\mu}\left(x_{1}, x_{p}\right)=+\infty$. We claim that there exists $i_{0}$ such that $\Lambda_{\lambda}\left(x_{i_{0}}, x_{i_{0}+1}\right)=+\infty$. Assume that $\Lambda_{\lambda}\left(x_{i}, x_{i+1}\right)<+\infty$ for all $i=1, \ldots, p-1$. Using (8) and part (iii) of Proposition 3.3, it follows that $\Lambda_{\mu}\left(x_{1}, x_{p}\right)<+\infty$. This contradiction says that there exists $i_{0}$ such that $\Lambda_{\lambda}\left(x_{i_{0}}, x_{i_{0}+1}\right)=+\infty$. In this case, the inequality (6) holds true.

- Suppose that $\Lambda_{\mu}\left(x_{1}, x_{p}\right)<+\infty$. We also consider the following cases.

- If there exists $i_{0}$ such that $\Lambda_{\lambda}\left(x_{i_{0}}, x_{i_{0}+1}\right)=+\infty$, then the inequality (6) also holds true.

- We assume that $\Lambda_{\lambda}\left(x_{i}, x_{i+1}\right)<+\infty$ for all $i=1, \ldots, p-1$. Using (8) and part (iii) of Proposition 3.3 again, it follows that

$$
\Lambda_{\lambda}\left(x_{1}, x_{2}\right)+\Lambda_{\lambda}\left(x_{2}, x_{3}\right)+\cdots+\Lambda_{\lambda}\left(x_{p-1}, x_{p}\right)+(p-1) \epsilon \geq \Lambda_{\mu}\left(x_{1}, x_{p}\right) .
$$

By taking $\epsilon \rightarrow 0+$, we obtain the desired inequality (6).

Part (ii) is obvious. 
Proposition 3.6. Let $(X, M, *)$ be a rational fuzzy metric space such that the t-norm * is left-continuous at 1 with respect to the first or second component, and let $\left\{x_{n}\right\}_{n \in \mathbb{N}}$ be a sequence in $X$ such that there exist $0<k<1$ and $x^{*} \in X$ satisfying the following conditions:

- for all $n \in \mathbb{N}$, it holds

$$
M\left(x_{n}, x_{n+1}, t\right) \geq M\left(x^{*}, x_{1}, \frac{t}{k^{n}}\right) ;
$$

- given any $\lambda \in(0,1)$, there exists $t_{\lambda}>0$ such that $M\left(x^{*}, x_{1}, t_{\lambda}\right)>1-\lambda$ and $\sup _{\lambda \in(0,1)} t_{\lambda}<+\infty$.

Then $\left\{x_{n}\right\}_{n \in \mathbb{N}}$ is a Cauchy sequence.

Proof. Given $n \in \mathbb{N}$, let $\widehat{t}_{n, \lambda}=k^{n} \cdot t_{\lambda}$. From (9) and the second condition, we see that

$$
M\left(x_{n}, x_{n+1}, \widehat{t}_{n, \lambda}\right) \geq M\left(x^{*}, x_{1}, t_{\lambda}\right)>1-\lambda .
$$

Using the second property of part (iii) in Proposition 3.3, the inequality (10) says that

$$
\Lambda_{\lambda}\left(x_{n}, x_{n+1}\right) \leq \widehat{t}_{n, \lambda}=k^{n} \cdot t_{\lambda} \text { for all } n \in \mathbb{N} .
$$

Now we consider $m, n \in \mathbb{N}$ with $m>n$. Given any $\mu \in(0,1)$, using (11) and part (i) of Proposition 3.5, there exists $\lambda \in(0,1)$ (which depends on $m$ and $n$ ) such that

$$
\begin{aligned}
\Lambda_{\mu}\left(x_{m}, x_{n}\right) & \leq \Lambda_{\lambda}\left(x_{n}, x_{n+1}\right)+\Lambda_{\lambda}\left(x_{n+1}, x_{n+2}\right)+\cdots+\Lambda_{\lambda}\left(x_{m-1}, x_{m}\right) \\
& \leq k^{n} \cdot t_{\lambda}+k^{n+1} \cdot t_{\lambda}+\cdots+k^{m-1} \cdot t_{\lambda} \\
& =t_{\lambda} \cdot \frac{k^{n} \cdot\left(1-k^{m-n}\right)}{1-k} \leq t_{\lambda} \cdot \frac{k^{n}}{1-k} \\
& \leq\left(\sup _{\lambda \in(0,1)} t_{\lambda}\right) \cdot \frac{k^{n}}{1-k} \rightarrow 0 \text { as } n \rightarrow \infty,
\end{aligned}
$$

since $\sup _{\lambda \in(0,1)} t_{\lambda}<+\infty$ by the second condition, which shows that $\left\{x_{n}\right\}_{n \in \mathbb{N}}$ is a Cauchy sequence with respect to $\Lambda_{\mu}$ for all $\mu \in(0,1)$. Then, part (ii) of Proposition 3.4 completes the proof.

Let us recall that $M(x, y, t)$ can be interpreted as the membership degree of the distance that is less than or equal to $t$ between $x$ and $y$. Therefore, if the distance between $x$ and $y$ is finite, and $t_{x y}$ is a positive real number that is larger than the distance between $x$ and $y$, then, intuitively, we should have $M\left(x, y, t_{x y}\right)=1$, which means that the distance that is less than or equal to $t_{x y}$ between $x$ and $y$ is definitely true. The formal definition is given below.

Definition 3.3. Let $(X, M, *)$ be a fuzzy metric space. Given any two elements $x, y \in X$ with $x \neq y$, we say that $x$ and $y$ have a finite distance if and only if there exists $t_{x y}>0$ such that $M\left(x, y, t_{x y}\right)=1$. 
Corollary 3.1. Let $(X, M, *)$ be a rational fuzzy metric space such that the t-norm * is left-continuous at 1 with respect to the first or second component, and let $\left\{x_{n}\right\}_{n \in \mathbb{N}}$ be a sequence in $X$ such that there exist $0<k<1$ and $x^{*} \in X$ satisfying the following conditions:

- for all $n \in \mathbb{N}$, it holds

$$
M\left(x_{n}, x_{n+1}, t\right) \geq M\left(x^{*}, x_{1}, \frac{t}{k^{n}}\right) ;
$$

- $x^{*}$ and $x_{1}$ have a finite distance.

Then $\left\{x_{n}\right\}_{n \in \mathbb{N}}$ is a Cauchy sequence.

Proof. Since $x^{*}$ and $x_{1}$ have a finite distance, by definition, there exists $t^{*}>0$ such that $M\left(x, y, t^{*}\right)=1$. Therefore, given any $\lambda \in(0,1)$, there exists $t_{\lambda} \equiv t^{*}$ such that

$$
M\left(x^{*}, x_{1}, t_{\lambda}\right)=M\left(x^{*}, x_{1}, t^{*}\right)=1>1-\lambda \text { and } \sup _{\lambda \in(0,1)} t_{\lambda}=t^{*}<+\infty,
$$

which shows that the second condition of Proposition 3.6 is satisfied. This completes the proof.

As we mentioned before, Grabiec [5] provided a fixed point theorem in fuzzy metric spaces by defining a Cauchy sequence $\left\{x_{n}\right\}_{n \in \mathbb{N}}$ to be satisfying

$$
\lim _{n \rightarrow \infty} M\left(x_{n+p}, x_{n}, t\right)=1
$$

for all $p>0$ and $t>0$. In other words, the fixed point theorem in fuzzy metric spaces provided by Grabiec [5] is inappropriate. Here, we shall provide a fixed point theorem in fuzzy metric space based on the natural concept of Cauchy sequence in Definition 3.2.

Let $X$ be a universal set, and let $T: X \rightarrow X$ be a mapping from $X$ into itself. Given any initial point $x_{0} \in X$, we can construct a sequence $\left\{x_{n}\right\}_{n \in \mathbb{N}}$ defined by

$$
x_{n}=T\left(x_{n-1}\right)
$$

for $n=1,2, \ldots$. Then, we have

$$
x_{n}=T^{n}\left(x_{0}\right)
$$

for $n=1,2, \ldots$

Let $(X, d)$ be a metric space, and let $T: X \rightarrow X$ be a mapping from $X$ into itself. For $0<k<1$, we recall that $T$ is a contractive mapping according to $d$ with a contractive constant $k$ if

$$
d(T(x), T(y)) \leq k \cdot d(x, y) .
$$

We define $a * b=a \cdot b$ and

$$
M_{d}(x, y, t)=\frac{t}{t+d(x, y)} .
$$


Then, it is easy to check that $\left(X, M_{d}, *\right)$ is a fuzzy metric space induced by $d$. We have the following interesting properties.

Proposition 3.7. Let $\left(X, M_{d}, *\right)$ be a fuzzy metric space induced by a given metric space $(X, d)$, and let $T: X \rightarrow X$ be a mapping from $X$ into itself. If $T$ is a contractive mapping according to $d$ with a contractive constant $k$, then

$$
M_{d}(T(x), T(y), t) \geq M_{d}\left(x, y, \frac{t}{k}\right) .
$$

Proof. From (14), we have

$$
t+d(T(x), T(y)) \leq t+k \cdot d(x, y),
$$

which implies

$$
\frac{t}{t+d(T(x), T(y))} \geq \frac{t}{t+k \cdot d(x, y)}
$$

for $t>0$. Therefore we obtain

$$
\begin{aligned}
M_{d}(T(x), T(y), t) & =\frac{t}{t+d(T(x), T(y))} \\
& \geq \frac{t}{t+k \cdot d(x, y)}=\frac{\frac{t}{k}}{\frac{t}{k}+d(x, y)}=M_{d}\left(x, y, \frac{t}{k}\right) .
\end{aligned}
$$

This completes the proof.

Based on Proposition 3.7, we propose the following definitions.

Definition 3.4. Let $(X, M, *)$ be a fuzzy metric space, and let $T: X \rightarrow X$ be a mapping from $X$ into itself. We say that $T$ is a contractive mapping according to $M$ with a contractive constant $0<k<1$ if and only if, given any $x, y \in X$,

$$
M(T(x), T(y), t) \geq M\left(x, y, \frac{t}{k}\right)
$$

for all $t>0$.

Theorem 3.1 (Fixed Point Theorem). Let $(X, M, *)$ be a complete rational fuzzy metric space such that the $t$-norm * is left-continuous at 1 with respect to the first or second component. Let $T: X \rightarrow X$ be a contractive mapping according to $M$ with contractive constant $0<k<1$. Suppose that there exists an element $x^{*} \in X$ such that, given any $\lambda \in(0,1)$, there exists $t_{\lambda}>0$ satisfying $M\left(x^{*}, T\left(x^{*}\right), t_{\lambda}\right)>1-\lambda$ and $\sup _{\lambda \in(0,1)} t_{\lambda}<+\infty$. Then the following statements hold true.

(i) The mapping $T$ has a fixed point $x^{\circ}$ that is obtained by taking the limit $x_{n} \stackrel{M}{\longrightarrow} x^{\circ}$ as $n \rightarrow \infty$, where the sequence $\left\{x_{n}\right\}_{n \in \mathbb{N}}$ is constructed from the initial element $x_{0}=x^{*}$ according to (13).

(ii) If for any $x, y \in X$ with $x \neq y$, there exists $t_{0}>0$ such that $M\left(x, y, t_{0}\right)$ $\neq 0$, then the fixed point of $T$ is unique. 
Proof. From (15), by induction on $n$, we obtain

$$
M\left(x_{n}, x_{n+1}, t\right) \geq M\left(x_{0}, x_{1}, \frac{t}{k^{n}}\right)
$$

for all $t>0$ and $n \in \mathbb{N}$. To prove part (i), by taking the initial element $x_{0}=x^{*}$, using Proposition 3.6, it follows that $\left\{x_{n}\right\}_{n \in \mathbb{N}}$ is a Cauchy sequence. The completeness of $(X, M, *)$ says that there exists $x^{\circ} \in X$ such that $x_{n} \stackrel{M}{\longrightarrow} x^{\circ}$ as $n \rightarrow \infty$, i.e.,

$$
\lim _{n \rightarrow \infty} M\left(x_{n}, x^{\circ}, t\right)=1
$$

for all $t>0$. Since $M\left(x_{n}, x^{\circ}, t\right) \leq 1$, it also says that

$$
M\left(x_{n}, x^{\circ}, t\right) \rightarrow 1^{-} \text {as } n \rightarrow \infty .
$$

Now, by the triangle inequality, we have

$$
\begin{aligned}
M\left(T\left(x^{\circ}\right), x^{\circ}, t\right) & \geq M\left(T\left(x^{\circ}\right), T\left(x_{n}\right), \frac{t}{2}\right) * M\left(T\left(x_{n}\right), x^{\circ}, \frac{t}{2}\right) \\
& =M\left(T\left(x^{\circ}\right), T\left(x_{n}\right), \frac{t}{2}\right) * M\left(x_{n+1}, x^{\circ}, \frac{t}{2}\right) \\
& \geq M\left(x^{\circ}, x_{n}, \frac{t}{2 k}\right) * M\left(x_{n+1}, x^{\circ}, \frac{t}{2}\right),
\end{aligned}
$$

where the latter inequality follows from (15) and the monotonicity of a t-norm. According to (16) and applying the left-continuity of t-norm $*$ at 1 , we have

$$
M\left(x^{\circ}, x_{n}, \frac{t}{2 k}\right) * M\left(x_{n+1}, x^{\circ}, \frac{t}{2}\right) \rightarrow 1 * 1=1 \text { as } n \rightarrow \infty .
$$

Using (17) and (18), we obtain $M\left(T\left(x^{\circ}\right), x^{\circ}, t\right)=1$ for all $t>0$, which says that $T\left(x^{\circ}\right)=x^{\circ}$.

To prove part (ii), suppose there is another fixed point $y$, i.e., $T(y)=y$. Since $(X, M, *)$ is a rational fuzzy metric space, for $x \neq y$, we have

$$
\lim _{t \rightarrow 0+} M(x, y, t)=0 \text {. }
$$

Now, using (15), we can obtain

$$
\begin{aligned}
M\left(y, x^{\circ}, k^{n} t\right) & =M\left(T(y), T\left(x^{\circ}\right), k^{n} t\right) \\
& \geq M\left(y, x^{\circ}, k^{n-1} t\right) \\
& =M\left(T(y), T\left(x^{\circ}\right), k^{n-1} t\right) \\
& \geq M\left(y, x^{\circ}, k^{n-2} t\right) \\
& \geq \cdots \geq M\left(y, x^{\circ}, t\right) \geq 0 .
\end{aligned}
$$

Suppose that $y \neq x^{\circ}$. Since $0<k<1$, taking $n \rightarrow \infty$ and using (19), we obtain $M\left(y, x^{\circ}, t\right)=0$ for all $t>0$. By the extra assumption, there exists $t_{0}>0$ such that $M\left(y, x^{\circ}, t_{0}\right) \neq 0$. This contradiction says that we must have $y=x^{\circ}$, and the proof is complete. 
Since $M(x, y, t)$ is the membership degree of the distance that is less than or equal to $t$ between $x$ and $y$, intuitively, for any $x, y \in X$ with $x \neq y$, there exists a sufficiently small $t_{0}>0$ such that the distance between $x$ and $y$ is less than or equal to $t_{0}$, which also means $M\left(x, y, t_{0}\right)>0$. Therefore, the extra assumption in part (ii) of Theorem 3.1 for guaranteeing the uniqueness is reasonable.

Theorem 3.2 (Fixed Point Theorem). Let $(X, M, *)$ be a complete rational fuzzy metric space such that the $t$-norm $*$ is left-continuous at 1 with respect to the first or second component. Let $T: X \rightarrow X$ be a contractive mapping according to $M$ with contractive constant $0<k<1$. Suppose that $x$ and $y$ have a finite distance for any $x, y \in X$ with $x \neq y$. Then, the mapping $T$ has a unique fixed point $x^{\circ}$ that is obtained by taking the limit $x_{n} \stackrel{M}{\longrightarrow} x^{\circ}$ as $n \rightarrow \infty$, where the sequence $\left\{x_{n}\right\}_{n \in \mathbb{N}}$ is constructed from any initial element $x_{0} \in X$ according to (13).

Proof. Since $x$ and $y$ have a finite distance, the extra assumption in part (ii) of Theorem 3.1 is satisfied automatically. Therefore, the result follows from the same argument in the proof of Theorem 3.1 by using Corollary 3.1. This completes the proof.

\section{References}

[1] C. Alaca, D. Turkoglu, and C. Yildiz, Fixed points in intuitionistic fuzzy metric spaces, Chaos Solitons Fractals 29 (2006), no. 5, 1073-1078.

[2] S. S. Chang, Y. J. Cho, and S. M. Kang, Nonlinear operator theory in probabilistic metric space, Nova Science Publishers, New York, 2001.

[3] A. George and P. Veeramani, On some results in fuzzy metric spaces, Fuzzy Sets and Systems 64 (1994), no. 3, 395-399.

[4] - On some results of analysis for fuzzy metric spaces, Fuzzy Sets and Systems 90 (1997), no. 3, 365-368.

[5] M. Grabiec, Fixed points in fuzzy metric spaces, Fuzzy Sets and Systems 27 (1988), no. $3,385-389$.

[6] V. Gregori and S. Romaguera, On completion of fuzzy metric spaces, Fuzzy Sets and Systems 130 (2002), no. 3, 399-404.

[7] V. Gregori, S. Romaguera, and A. Sapena, A note on intuitionistic fuzzy metric spaces, Chaos Solitons Fractals 28 (2006), no. 4, 902-905.

[8] V. Gregori and A. Sapena, On fixed-point theorems in fuzzy metric spaces, Fuzzy Sets and Systems 125 (2002), no. 2, 245-252.

[9] O. Hadžić and E. Pap, Fixed Point Theory in Probabilistic Metric Spaces, Klumer Academic Publishers, 2001.

[10] O. Kaleva and S. Seikkala, On fuzzy metric spaces, Fuzzy Sets and Systems 12 (1984), no. 3, 215-229.

[11] I. Kramosil and J. Michalek, Fuzzy metric and statistical metric spaces, Kybernetika 11 (1975), no. 5, 336-344.

[12] B. Schweizer and A. Sklar, Statistical metric spaces, Pacific J. Math. 10 (1960), 313-334.

[13] - Triangle inequalities in a class of statistical metric spaces, J. London Math. Soc. 38 (1963), 401-406.

[14] B. Schweizer, A. Sklar, and E. Thorp, The metrization of statistical metric spaces, Pacific J. Math. 10 (1960), 673-675. 
[15] S. Sharma and B. Deshpande, Common fixed point theorems for finite numbers of mappings without continuity and compatibility on intuitionistic fuzzy metric spaces, Chaos Solitons Fractals 40 (2009), no. 5, 2242-2256.

[16] G. Song, Comments on "A common fixed point theorem in a fuzzy metric space", Fuzzy Sets and Systems 135 (2003), no. 3, 409-413.

[17] P. V. Subrahmanyam, A common fixed point theorem in fuzzy metric spaces, Inform. Sci. 83 (1995), no. 3-4, 109-112.

[18] R. Vasuki and P. Veeramani, Fixed point theorems and cauchy sequences in fuzzy metric spaces, Fuzzy Sets and Systems 135 (2003), no. 3, 415-417.

Department of Mathematics

National Kaohsiung Normal University

KAOHSIUNG 802, TAIWAN

E-mail address: hcwu@nknucc.nknu.edu.tw 